\title{
Erratum to: Inexpressible properties and Grelling's antinomy
}

\section{Benjamin Schnieder}

\section{Erratum to: Philos Stud (2010) 148:369-385 \\ DOI 10.1007/s11098-008-9329-7}

Unfortunately, negation signs are missing throughout the article. The following passages are affected:

Page 374

Barber $_{\leftrightarrow}$ The said barber shaves himself $\leftrightarrow$ the said barber shaves himself, should read

Barber $_{\leftrightarrow}$ The said barber shaves himself $\leftrightarrow \neg$ the said barber shaves himself, Page 374

Set $\leftrightarrow$ The said set contains itself $\leftrightarrow$ the said set contains itself, should read

Set $\leftrightarrow \quad$ The said set contains itself $\leftrightarrow \neg$ the said set contains itself, Page 377

Predicate $_{\leftrightarrow}$ The said predicate applies to itself $\leftrightarrow$ the said predicate applies to itself.

The online version of the original article can be found under doi:10.1007/s11098-008-9329-7.

B. Schnieder $(\bowtie)$

Institut für Philosophie, Humboldt-Universität zu Berlin, Unter den Linden 6, Berlin 10099, Germany

e-mail: b.schnieder@gmx.de 
should read

Predicate $_{\leftrightarrow}$ The said predicate applies to itself $\leftrightarrow \neg$ the said predicate applies to itself.

Page 383

The derivation should read:

\begin{tabular}{llll}
\hline 1 & $(1)$ & $P^{*}$ exists & A \\
2 & $(2)$ & $t$ expresses $P^{*}$ & A \\
$2, \mathrm{Ex}$ & $(3)$ & $\forall \mathrm{x}\left(t\right.$ applies to $\mathrm{x} \leftrightarrow \mathrm{x}$ has $\left.P^{*}\right)$ & $2, \mathrm{Ex}$ \\
$1, \mathrm{DN}$ & $(4)$ & $\forall \mathrm{x}\left(\mathrm{x}\right.$ has $P^{*} \leftrightarrow \neg \mathrm{x}$ applies to $\left.\mathrm{x}\right)$ & $1, \mathrm{DN}$ \\
$1,2, \mathrm{DN}, \mathrm{Ex}$ & $(5)$ & $\forall \mathrm{x}(t$ applies to $\mathrm{x} \leftrightarrow \neg \mathrm{x}$ applies to $\mathrm{x})$ & $3,4 \mathrm{FOPL}$ \\
$1,2, \mathrm{DN}, \mathrm{Ex}$ & $(6)$ & $t$ applies to $t \leftrightarrow \neg t$ applies to $t$ & $4 \forall \mathrm{E}$ \\
$1, \mathrm{DN}, \mathrm{Ex}$ & $(7)$ & $\neg t$ expresses $P^{*}$ & 6,2 FOPL (RAA) \\
$1, \mathrm{DN}, \mathrm{Ex}$ & $(8)$ & $\forall \mathrm{y} \neg \mathrm{y}$ expresses $P^{*}$ & $6, \forall \mathrm{I}$ \\
\hline
\end{tabular}

\section{Page 384}

The derivation should read:

\begin{tabular}{llll}
\hline 1 & $(1)$ & $a$ is a member of $S$ & A \\
2 & $(2)$ & $\forall \mathrm{y}(\mathrm{y}$ is a member of $S \rightarrow(a$ bears $R$ to $y \leftrightarrow \neg y$ bears $R$ to $y))$ & A \\
3 & $(3)$ & $\forall \mathrm{x}(\mathrm{x}$ is a member of $S \rightarrow(x$ bears $R$ to $x \vee \neg x$ bears $R$ to $x))$ & A \\
1,2 & $(4)$ & $a$ bears $R$ to $a \leftrightarrow \neg a$ bears $R$ to $a$ & $2 \forall \mathrm{E} ; 1,2$ MPP \\
1,3 & $(5)$ & $a$ bears $R$ to $a \vee \neg a$ bears $R$ to $a$ & $3 \forall \mathrm{E}$; 1,3 MPP \\
6 & $(6)$ & $a$ bears $R$ to $a$ & A \\
$1,2,6$ & $(7)$ & $\neg a$ bears $R$ to $a$ & $6,4 \leftrightarrow \mathrm{E}$ \\
$1,2,6$ & $(8)$ & $a$ bears $R$ to $a \& \neg a$ bears $R$ to $a$ & $7,8 \& \mathrm{I}$ \\
9 & $(9)$ & $\neg a$ bears $R$ to $a$ & A \\
$1,2,9$ & $(10)$ & $a$ bears $R$ to $a$ & $9,4 \leftrightarrow \mathrm{E}$ \\
$1,2,9$ & $(11)$ & $a$ bears $R$ to $a \& \neg a$ bears $R$ to $a$ & $9,10 \& \mathrm{I}$ \\
$1,2,3$ & $(12)$ & $a$ bears $R$ to $a \& \neg a$ bears $R$ to $a$ & $5,6,8,9,11 \vee \mathrm{E}$ \\
\hline
\end{tabular}

